\title{
Spectroelectrochemical Investigation of 4-Dicyanomethylene 2,6-Dimethyl-4H-Pyran (DDP) Dye with Guanidine Hydrochloride $(\mathrm{GuHCl})$ in Water
}

\author{
Somasundaram Gayathri ${ }^{1}$, Rajaraman Vasanthi ${ }^{1}$, Mahalingam Vanjinathan ${ }^{1}$, \& Rajendran Kumaran ${ }^{1}$ \\ ${ }^{1}$ Department of Chemistry, Dwaraka Doss Goverdhan Doss Vaishnav College (Autonomous), Gokul Bagh, \\ E.V.R. Periyar Road, Arumbakkam, Chennai, Tamil Nadu, India \\ Correspondence: Rajendran Kumaran, Department of Chemistry, Dwaraka Doss Goverdhan Doss Vaishnav \\ College (Autonomous), 833, Gokul Bagh, E.V.R. Periyar Road, Arumbakkam, Chennai 600 106, Tamil Nadu, \\ India. Tel: 091-44-2475-6655. E-mail: kumaranwau@rediffmail.com
}

Received: June 1, 2019

doi:10.5539/jmsr.v8n3p6
Accepted: July 5, 2019

Online Published: July 15, 2019

URL: https://doi.org/10.5539/jmsr.v8n3p6

\begin{abstract}
Interaction of guanidine hydrochloride $(\mathrm{GuHCl})$ with 4-dicyanomethylene-2,6-dimethyl-4H-pyran (DDP) dye were investigated by photophysical and electrochemical techniques. An enhancement in the fluorescence intensity and formation of an isosbestic point revels that the ground state and excited state properties of DDP dye. The nature of interaction between DDP dye and $\mathrm{GuHCl}$ is predominantly through hydrogen-bonding even thoughelectrostatic interaction in aqueous phase operate. The existence of electrostatic interaction by current response function describes that the shift in anodic peak potential towards less positive value indicate electrostatic binding. The existence of more than one microenvironment of DDP dye in aqueous phase is elucidated by Electrochemical Impedence Spectroscopy (EIS) studies through Nyquist plots. These plots signify that there exist at least three different micro environments or dye existing in different fluorescence lifetimes with varying of amplitude. Fluorescence spectral techniques along with electrochemical studies are used as efficient tools to elucidate the nature of interaction of a water soluble probe with hydrogen-bonding solutes is elucidated in our study.
\end{abstract}

Keywords: DDP Dyes, Guanidine Hydrochloride, Hydrogen-Bonding, Fluorescence Emission, Fluorescence Lifetime, Cyclic Voltammetry, Electrochemical Impedance Spectroscopy

\section{Introduction}

4-Dicyanomethylene-2,6-dimethyl-4H-pyran (DDP) dye is a water soluble fluorophore, that exhibit electroluminescent properties also. The structure of DDP dye is similar to that of DCM type dye (Woods, 1958; Hammond, 1979; Meyer \& Mialocq, 1987; Hsieh \& Chen, 2007; Zarins, Jubels, \& Kokars, 2011) which is a well known ICT type dye.DDP dye has a strong acceptor group (dicyanomethylene $-\mathrm{C}(\mathrm{CN})_{2}$ ) in $4^{\text {th }}$ position and a donor moiety (methyl) substituted in $2^{\text {nd }}$ and $6^{\text {th }}$ positions. DDP dye possesses a significant advantage over DCM dye due to its better solubility in water and its photophysical property is distinctly different from DCM dye. There is no possibility of Twisted Intramolecular Charge Transfer (TICT) phenomenon unlike DCM dye such that the complexity over DCM dye is completely eliminated.Further, the significant edge over DCM dye is that the DDP dyeexhibits a single broad emission maxima in the visible regionwhereas DCM dye results in abroader spectra very near to the NIR region accompanied with dual emissive character.As reported in the literature pyranylidene derivatives as fluorophores are widely applied as coating materials in OLED substances and they play a major role as dopant in a polymer matrix at limited concentration which is of importance in the field of optoelectronics. Red-emitter pyranylidene-type compound usually have high luminescence quantum yield but their chromaticity is less in the presence of mono electron donor substituents (Zarins, Jubels, \& Kokars, 2011). On the contrary, DDP dye exhibit excellent luminescence character in the presence of more electron releasing moieties and acts as an ideal is used as the host molecule. The present study involves Guanidine Hydrochloride $(\mathrm{GuHCl})$ as the guest molecule and the role of $\mathrm{GuHCl}$ on the photophysical and electrochemical properties of DDP dye is established using fluorescence approach assisted by impedance spectral studies.

The interaction of water soluble dyes exhibiting Intramolecular Charge Transfer (ICT) and Photoinduced 
Electron Transfer (PET) with non-fluorescent hydrogen-bonding solutes like urea derivatives, amides, GuHCl and cyclodextrins has been well established in the literature.(Srividya, Ramamurthy, Shanmugasundaram, \& Ramakrishna, 1996; Kumaran \& Ramamurthy, 2006; Kumaran \& Ramamurthy, 2010; Kumaran, Varalakshmi, \& Padma Malar, 2010; Gayathri, Vasanthi, Vanjinathan, \& Kumaran, 2018; Sowntharya, Gayathri, Dhenadayalan, Vasanthi, Vanjinathan, \& Kumaran, 2017) These studies provides an in-depth understanding on the photophysical properties of host-guest systems. A recent report on DDP dye interaction with amides (Gayathri, Vasanthi, Vanjinathan, \& Kumaran, 2018) and urea derivatives (Kumaran \& Ramamurthy, 2006) signifies that hydrogen-bonding interactions accompanied with hydrophobicity of the solutes influences the fluorescence emission, lifetime and current-potential voltammograms. The concentration, nature of the solute and the presence of hydrogen-bonding or hydrophobic moieties results in the formation of several microenvironments in aqueous solution such that the dye resides in multi heterogeneous arrangement in aqueous solution with large variation in their fluorescence lifetimes and relative amplitude.

$\mathrm{GuHCl}$ is a non-fluorescent solute and has excellent solubility in water similar to that of urea but differs from urea on the basis of hydrogen-bonding abilities. $\mathrm{GuHCl}$ forms hydrogen-bonding with water molecule through $\mathrm{N}-\mathrm{H}$ hydrogen and acts as a hydrogen-bonding donor only wherein urea acts as a hydrogen-bonding donor as well as an acceptor (Kawahara \& Tanford, 1966). It has been established that $\mathrm{GuHCl}$ is surrounded approximately by 12 to 13 water molecules and six water molecules are involved in a direct hydrogen-bonding interaction with GuHCl (Castellino \& Barker, 1968; Garza-Ramos et al., 1992; Strambini \& Gonnelli, 1986; Ma \& Tsou, 1991; Miggiano, Mordente, Pischiutta, Martorana, \& Castelli, 1987; Zettl meissl, Rudolph, \& Jaenicke, 1982; Moosavi-Movahedi, Naderi, \& Farzami, 1994; Morjana, McKeone, \& Gilbert, 1993; Shoshani, Darszon, Tijena de Gomez-puyou, \& Gomez-puyou, 1994; Cox, 1968; Katz, 1968; Makhatadze \& Privalov, 1992; Hedwig, Lilley, \& Linsdell, 1991; Michnik \& Sulkowska, 1997). This variation in the hydrogen-bonding pattern compared to urea is significantly important because the nature and the type of hydrogen-bonding arrangement influence the photophysical properties of fluorescence probes. An increase in the concentration of GuHCl results in the partial displacement of the water molecules from the secondary hydration sphere resulting in a GuHCl-water hydrogen-bonding network throughout the medium. This results in a large variation in the orientation of the hydrophobic and hydrophilic clusters in aqueous phase in the close vicinity of $\mathrm{GuHCl}$ (Castellino \& Barker, 1968; Moosavi-Movahedi, Naderi, \& Farzami, 1994; Morjana, McKeone, \& Gilbert, 1993; Shoshani, Darszon, Tijena de Gomez-puyou, \& Gomez-puyou, 1994; Cox, 1968).

$\mathrm{GuHCl}$ forms molecular cages of extended hydrogen-bonded network with water molecules, clusters and self assemblies (Michnik \& Sulkowska, 1997) in hydrogen-bonding solvents. $\mathrm{GuHCl}(6.0 \mathrm{M})$ exhibit large viscosity and density, which is of significant importance in the concept of photophysical properties of fluorescence probes. $\mathrm{GuHCl}$ possesses a very high ionic strength in water and acts as a strong electrolyte (Moosavi-Movahedi, Naderi, \& Farzami, 1994). The presence of positive charge on the imino nitrogen in $\mathrm{GuHCl}$ results in an unusual structure compared to urea. The presence of an imine and aminoacetal functional group in $\mathrm{GuHCl}$ result in a large variation in the hydrogen-bonding properties (number of hydrogen-bonding acceptor and donor groups) compared to that of urea in aqueous solutions. Apart from the solvent mediated hydrogen-bonding interactions, the electrostatic interactions in combination with hydrogen-bonding influence the excited state properties of probes. $\mathrm{GuHCl}$ and urea both possess strong hydrogen-bonding properties with solvents and have a tendency to alter the structure of the water molecules which is crucial in biological studies concerned to $\mathrm{GuHCl}$ (Strambini \& Gonnelli, 1986; Zettl meissl, Rudolph, \& Jaenicke, 1982; Moosavi-Movahedi, Naderi, \& Farzami, 1994; Morjana, McKeone, \& Gilbert, 1993; Shoshani, Darszon, Tijena de Gomez-puyou, \& Gomez-puyou, 1994; Cox, 1968; Katz, 1968; Makhatadze \& Privalov, 1992; Hedwig, Lilley, \& Linsdell, 1991). The variation in the photophysical properties of probes with strong chaotropic agents is an area of unexplored domain and the literature regarding the photophysical properties of an extrinsic fluorescent probe with $\mathrm{GuHCl}$ is reported less in literature compared to that of urea interaction with fluorescent probes in aqueous solution (Kumaran, Varalakshmi, \& Padma Malar, 2010; Sowntharya, Gayathri, Dhenadayalan, Vasanthi, Vanjinathan, \& Kumaran, 2017; Kawahara \& Tanford, 1966; Castellino \& Barker, 1968; Garza-Ramos et al., 1992; Strambini \& Gonnelli, 1986). A significant change in the microenvironment of the aqueous phase and a large variation in the hydrogen-bonding properties are promoted on the further increase in the number of $\mathrm{GuHCl}$ molecules (Kawahara \& Tanford, 1966; Castellino \& Barker, 1968; Michnik \& Sulkowska, 1997). The present study provides an insight on the variation in the photophysical properties of DDP dye with $\mathrm{GuHCl}$ in comparison with amides and urea derivatives in aqueous solution since most of the biophysical and biochemical studies of the microheterogeneous systems involve the changes at the microscopic level.

Fluorescence techniques coupled with electrochemical methods are the most widely applicable tool to elucidate 
the nature of interaction between a fluorescent probes (DDP) with GuHCl. The Binding constant (K) and Free energy $(\Delta \mathrm{G})$ are determined for DDP-GuHCl from Cyclic Voltammetry (CV) and the presence of multi environment existing in aqueous solution is ascertained through Nyquist Plots obtained from Electrochemical Impedance Spectral (EIS) studies.

\section{Experimental}

\subsection{Materials}

GuHCl 2,6-dimethyl-4-pyrone, malononitrile, acetic anhydride, and potassium chloride were obtained from Merck chemicals. The solution of $\mathrm{GuHCl}$ was prepared using triple distilled water and the bulk concentration of $\mathrm{GuHCl}$ was restricted up to $6.0 \mathrm{M}$. The concentration of DDP was fixed at $7.1 \times 10^{-4} \mathrm{M}$ and was diluted with different concentration of $\mathrm{GuHCl}$ for photophysical studies. The DDP dye was synthesized based on the literature reported (Gayathri, Vasanthi, Vanjinathan, \& Kumaran, 2018).

\subsection{Experimental Techniques}

\subsubsection{Steady-State Absorption and Fluorescence Measurements}

Absorption spectra were recorded using an Agilent 8453 UV-Visible diode array spectrophotometer. The lamp source used for the ultraviolet-visible range was deuterium and tungsten lamps respectively. Absorption spectrum was recorded using water as the blank solution and the spectral range was from 190-750nm. Emission spectra and 3D spectral measurements were recorded in Fluoromax-4P spectrofluorimeter (Horiba JobinYvon) using fluorescence software provided by the manufacturer. The emission maximum corresponding to each excitation wavelength was recorded and a plot was generated using Origin 7.1 Software.

\subsubsection{Time-Resolved Fluorescence Measurements- Time Correlated Single-Photon Counting (TCSPC)} Technique

The Fluorescence decay measurements of DDP dye with GuHCl were recorded using IBH time correlated single-photon counting spectrometer with micro channel plate photomultiplier tube (MCP-PMT) (Hamamatsu, R3809U) as detector. The data analysis was carried out by the software provided by IBH (DAS-6) which is based on reconvolution technique using iterative non-linear least square methods (Gayathri, Vasanthi, Vanjinathan, \& Kumaran, 2018). A detailed experimental setup and analysis is provided in the supporting information.

\subsubsection{Cyclic Voltammetry (CV)}

CV and EIS studies were recorded using CH INSTRUMENT CH1604E electrochemical analyzer. The three electrode system consisted of the platinum electrode (surface area of $0.0314 \mathrm{~cm}^{2}$ ) as working electrode, $\mathrm{Ag} / \mathrm{AgCl}$ as the reference electrode and a platinum wire as the auxiliary electrode. Supporting electrolyte $(0.01 \mathrm{M} \mathrm{KCl})$ was used in all our electrochemical for recording voltammogram. The Voltammograms of DDP dye $\left(7.1 \times 10^{-4} \mathrm{M}\right)$ with varying concentration of $\mathrm{GuHCl}$ were recorded at scan rates ranging from $50 \geq v \leq 500 \mathrm{mVs}^{-1}$.

\subsubsection{Electrochemical Impedance Spectroscopy (EIS)}

EIS study is also known as alternate current (AC) conductivity study. Aplot of z' vs z" (Nyquist or cole-cole plots) taken over a wide frequency range $(50 \mathrm{~Hz}$ to $5 \mathrm{MHz})$ at room temperature was carried out. AC conductivity study is significant in understanding the formation of heterogeneous environment or accumulation of charges in the medium containing more than one micro environment (Gayathri, Vasanthi, Vanjinathan, \& Kumaran, 2018).

\section{Result and Discussion}

\subsection{Absorption Spectral Studies}

DDP dye exhibits two absorption peaks at 248 and $348 \mathrm{~nm}$ accompanied with a shoulder at $360 \mathrm{~nm}$ in water (Inset of Figure 1) as reported (Gayathri, Vasanthi, Vanjinathan, \& Kumaran, 2018). The absorption peak at $248 \mathrm{~nm}$ is attributed to the $\pi-\pi^{*}$ transition and the absorption peaks above $340 \mathrm{~nm}$ in water are attributed to the charge transfer (CT) bands. Addition of $\mathrm{GuHCl}$ results in an increase in the absorbance at the dye absorption maximum accompanied with a formation of isosbestic points at 300 and 370nm (Figure 1). The CT absorption maximum of DDP dye is influenced by the presence of large number of $\mathrm{GuHCl}$ molecules and the presence of isosbestic points signify a ground state complex formation between dye and $\mathrm{GuHCl}$. The nature of interaction of DDP-GuHCl could not be clearly ascertained from the absorption spectra due to the close spacing of the energy levels in the ground state. A similar behaviour was also observed in the interaction of DDP dye in the presence of formamide and alkyl substituted amides (Gayathri, Vasanthi, Vanjinathan, \& Kumaran, 2018). In order to establish the role of $\mathrm{GuHCl}$ and the influence on the excited state properties of DDP dye, steady state emission and time resolved fluorescence spectral studies were carried out. 


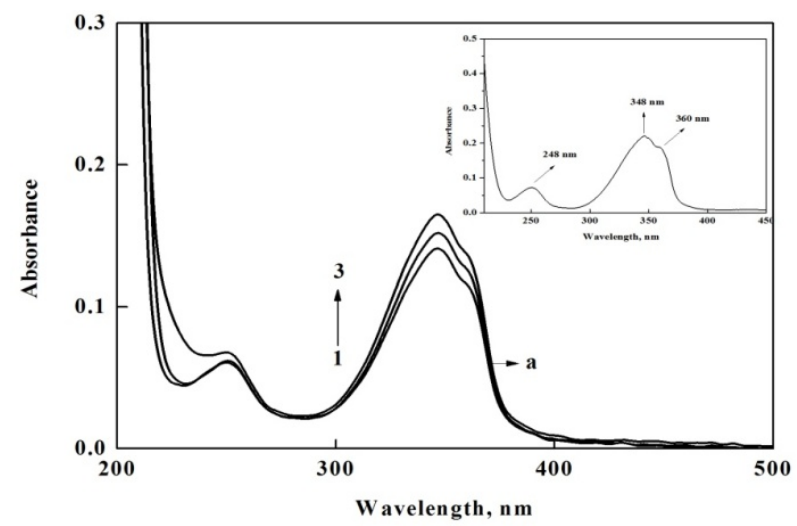

Figure 1. Absorption spectra of DDP dye $\left(7.1 \times 10^{-5}\right)$ with $\mathrm{GuHCl}$ in water. 1)DDP dye $+1.0 \mathrm{M} \mathrm{GuHCl}$, 2) DDP dye $+2.0 \mathrm{M} \mathrm{GuHCl}, 3) \mathrm{DDP}$ dye $+3.0 \mathrm{M} \mathrm{GuHCl}$. (a) Isosbestic point. (Inset Figure :UV-Visible absorption Spectrum of $7.1 \times 10^{-5} \mathrm{M}$ DDP dye)

\subsection{Fluorescence Studies}

DDP dye exhibits emission maximum around $430 \pm 2 \mathrm{~nm}$. Addition of $\mathrm{GuHCl}$ to DDP dye results in a gradual increase in the fluorescence intensity accompanied with a red shift in the emission maximum (inset of Figure 2). The extent of fluorescence enhancement is 3.0 fold $(\mathrm{GuHCl} 5.0 \mathrm{M})$ as shown in Figure 3 and the shift in emission maxima is shown in Figure 2. The variation in the emission spectral studies implies that the addition of $\mathrm{GuHCl}$ presumably influences the dipole moment of the medium.It is well known that addition of $\mathrm{GuHCl}$ increases the excited state dipole moment of water and results in a spectral shift towards the red region (Kumaran, Varalakshmi, \& Padma Malar, 2010). Further, the shift in the emission maximum towards the red region is presumably attributed to the presence of hydrogen-bonding interaction between $\mathrm{GuHCl}$ with DDP dye.

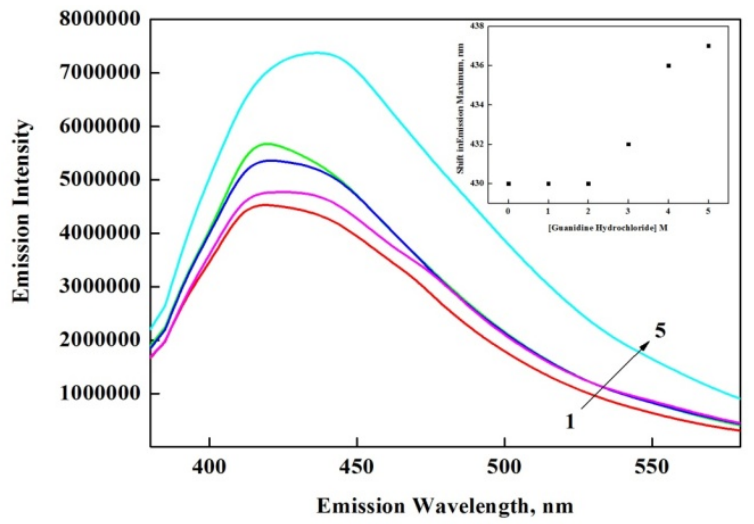

Figure 2. Emission spectra of $7.1 \times 10^{-5} \mathrm{M}$ DDP dye with $\mathrm{GuHCl}$ in water. 1) DDP dye, 2) DDP dye $+1.0 \mathrm{M}$ GuHCl, 3) DDP dye +2.0M GuHCl, 4) DDP dye +3.0M GuHCl, 5) DDP dye + 4.0M GuHCl, 6) DDP dye + $5.0 \mathrm{M} \mathrm{GuHCl}$. (Inset Figure: Shift of emission maximum of DDP dye with $\mathrm{GuHCl}$ in water)

In our earlier studies on interaction of urea with DDP dye also resulted in a shift in the emission maximum accompanied with a fluorescence enhancement. It is well known that the nature of the medium, presence of hydrophobic and hydrogen-bonding moieties of the solute molecules, the fluorescence intensity and emission maxima or dye molecules either increases or decreases. In the present study, microenvironment of DDP dye is definitely governed by addition of more number of solute particles in aqueous solution, which forms an extensive hydrogen-bonding network.

In order to establish the existence of hydrogen-bonding interaction of DDP-GuHCl complex, Benesi-Hildebrand plot (Benesi \& Hildebrand, 1949) was employed. Based on the emission intensity of DDP dye in the absence and presence of varying concentration of $\mathrm{GuHCl}$ (Figure 4), a plot was consulted. Interestingly, a non-linear fit was obtained and the plot reveals that $\mathrm{GuHCl}$ does not form any true complex with dye but it exhibits more than one 
binding sites of DDP dye with GuHCl. A true binding pattern was not observed at low or at high concentration of $\mathrm{GuHCl}$ which authenticates that there exists no 1:1 nor 1:2 binding pattern of DDP: GuHCl. Further, a modified plot was also constructed considering $\mathrm{GuHCl}$ upto $3.0 \mathrm{M}$ in order to obtain any binding pattern. A non-linear fit only (inset of Figure 4) was resulted even in the presence of less number of solute molecules.

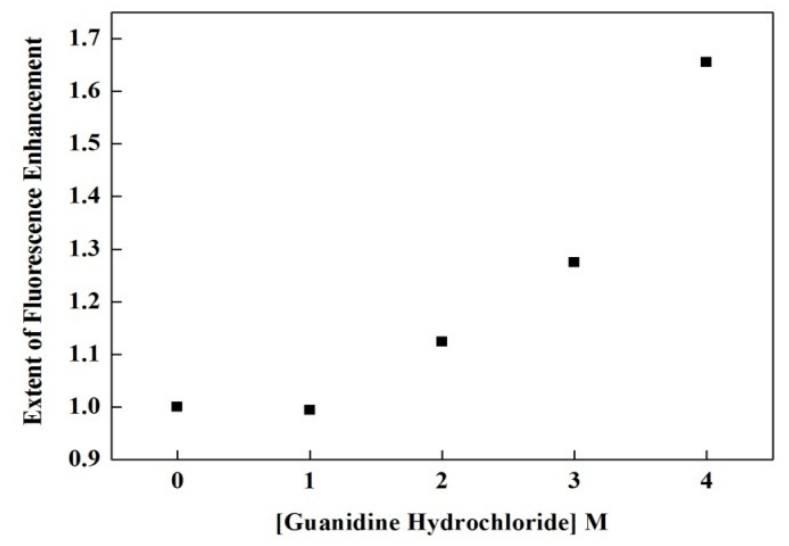

Figure 3. Extent of fluorescence enhancement of DDP dye with GuHCl

The regression co-efficient $\left(\mathrm{R}^{2}\right)$ value was found to be 0.98384 and 0.9641 at higher and lower concentration of $\mathrm{GuHCl}$ repectively, wherein the binding constant value was found to be very less compared to any other host-guest systems. A non-linear fit clearly indicates that there does not exists any specific 1:1 or 1:2 ratios or any other well defined ratio of DDP dye: $\mathrm{GuHCl}$ in aqueous solution. The number of $\mathrm{GuHCl}$ molecules surrounding the dye and water molecules does not follow any uniform pattern and hence no clear illustration about the orientation of dye surrounded by $\mathrm{GuHCl}$ could be established. Free energy $(-\Delta \mathrm{G})$ and binding constant $\mathrm{K}_{\mathrm{b}}$ calculated from steady state emission were found to be $15.41 \times 10^{3} \mathrm{KJ} \cdot \mathrm{mol}^{-1}$ and $2.16 \times 10^{-4} \mathrm{~mol} \mathrm{~L}^{-1}$ respectively. The existence of more than one micro environment in aqueous solution was clearly established from the presence of more than one fluorescence lifetime component in varying amplitude distribution.

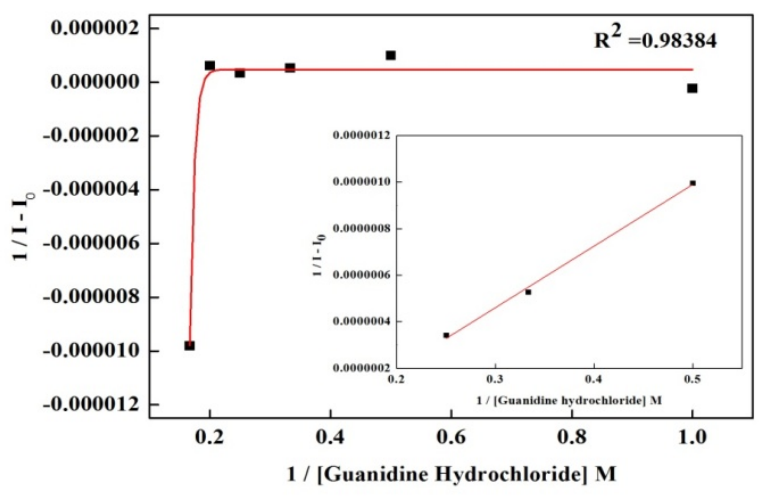

Figure 4. Benesi-Hildebrand Plot of DDP dye with GuHCl. (Inset Figure: Benesi-Hildebrand Plot of DDP dye with $\mathrm{GuHCl}$ at low concentration $1.0 \mathrm{M}$ to $3.0 \mathrm{M}$ )

\subsection{Time Resolved Fluorescence Studies}

The fluorescence lifetime decay of DDP dye exhibits a triexponential lifetime with three distinguishable microenvironments in the aqueous medium (Gayathri, Vasanthi, Vanjinathan, \& Kumaran, 2018). This was also correlated to the existence of three different layers in aqueous phase was authenticated from EIS studies (Figure 8). A short lifetime component of $0.2 \mathrm{~ns}$ accompanied with a relatively long lifetime component coexist in aqueous solution at any concentration of the $\mathrm{GuHCl}$ is observed. The addition of $\mathrm{GuHCl}$ results in the stabilization of three lifetime components irrespective of concentration of the $\mathrm{GuHCl}$. The fluorescence decay of $\mathrm{DDP}$ dye with $\mathrm{GuHCl}$ is shown in the Figure 5 and the fluorescence lifetime data is given in Table 1. On the 
addition of $\mathrm{GuHCl}$ there is no abrupt change in the relative amplitude distribution of these components but a significant decrease in the fluorescence lifetime of one component is visualised. The coexistence of three different lifetime components provides a complete orientation of DDP dye situated and oriented in different micro domains.

\subsubsection{GuHCl-DDP Dye Hydrogen-Bonding in the Presence of Water Molecules}

$\mathrm{GuHCl}$ forms a well organized hydrogen-bonding network with water molecules and in the absence of $\mathrm{GuHCl}$, the dye is surrounded by water molecules only. The microenvironment of dye is unaltered to a certain extent, even on the addition of very high concentration of $\mathrm{GuHCl}$. This was established based on the fluorescence lifetime data only. Approximately six to seven solvent molecules are involved in a direct hydrogen-bonding interaction with one $\mathrm{GuHCl}$ molecule and it forms self assembled clusters throughout the medium. A uniform hydrogen-bonding pattern is confined throughout the phase which results in organized self assembled clusters wherein the microenvironment is found to be the same. The interaction between the dye and $\mathrm{GuHCl}$ is hydrophobic in nature, and this is more pronounced in water. The interaction of water molecules through oxygen with any of the $-\mathrm{N}-\mathrm{H}$ moiety of $\mathrm{GuHCl}$ ion gives almost the same interaction energy. It is known that six water molecules are accommodated in each of the primary hydration sphere as well as in the secondary hydration sphere (Kawahara \& Tanford, 1966; Castellino \& Barker, 1968). An increase in the concentration of GuHCl results in the partial displacement of the water molecules from the secondary hydration sphere resulting in a $\mathrm{GuHCl}$-water- $\mathrm{GuHCl}$ hydrogen-bonding network throughout the medium. This results in a large variation in the orientation of the hydrophobic and hydrophilic clusters in aqueous phase in the close vicinity of $\mathrm{GuHCl}$ (Hedwig, Lilley, \& Linsdell, 1991; Michnik \& Sulkowska, 1997). A significant change in the microenvironment of the aqueous phase and a large variation in the hydrogen-bonding properties are promoted on the addition of $\mathrm{GuHCl}$, which is very similar to that of urea (Kumaran, Varalakshmi, \& Padma Malar, 2010). A detailed and schematic representation of GuHCl-DDP dye- water arrangement is provided in scheme 1.

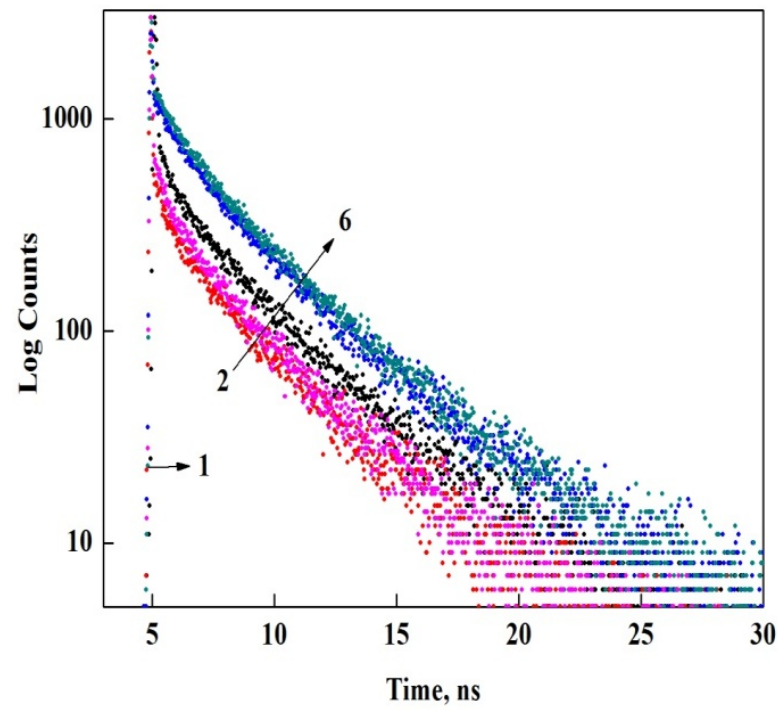

Figure 5. Fluorescence decay of DDP dye $\left(7.1 \times 10^{-5} \mathrm{M}\right)$ as a function of $\mathrm{GuHCl}$ in water. $\lambda_{\mathrm{ex}} 375 \mathrm{~nm}$ and $\lambda_{\mathrm{em}}$ 436nm. 1)Laser profile. 2) DDP dye alone, 3) DDP dye + GuHCl 2.0M, 4) DDP dye + GuHCl 3.0M, 5) DDP dye $+\mathrm{GuHCl}$ 4.0M, 6) DDP dye $+\mathrm{GuHCl} 6.0 \mathrm{M}$

The probabilistic arrangement was correlated based on the existence of the picosecond fluorescence lifetime component of DDP dye in presence of high concentration of $\mathrm{GuHCl}(6.0 \mathrm{M})$. We postulated a scheme wherein the formation of uniform $\mathrm{GuHCl}$-water hydrogen-bonding existing in throughout the medium and the introduction of the dye results in a formation of several new microenvironment containingcospheres containing GuHCl-water-dye hydrogen-bonding network such that dye-GuHC1 interactions predominate over GuHCl-water hydrogen-bonding network. 


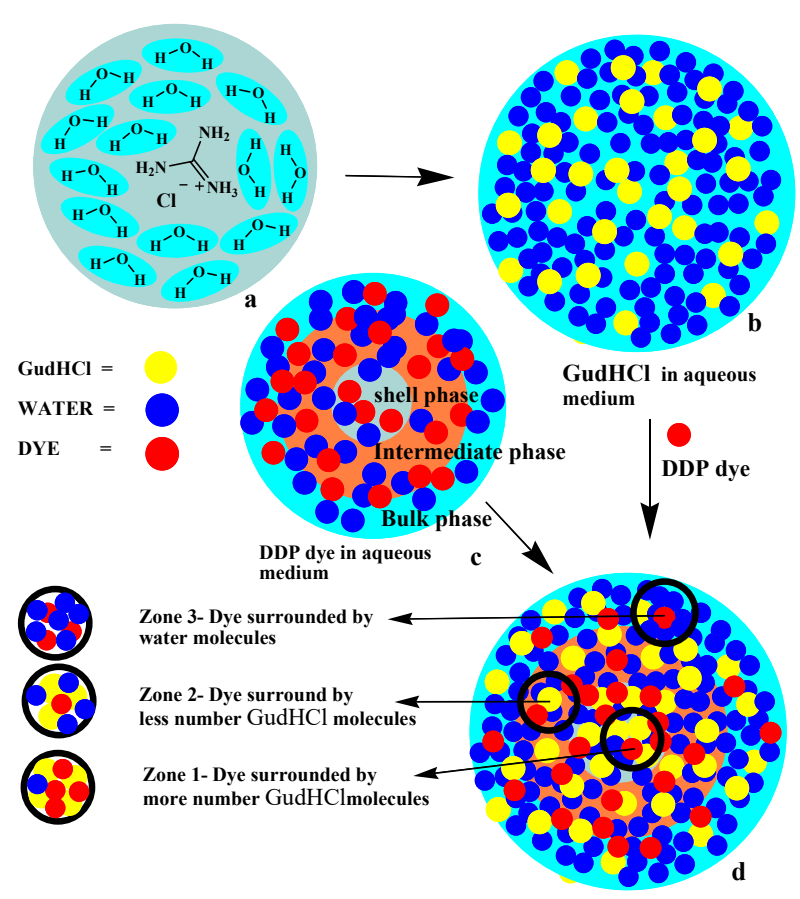

Scheme 1. Hydrogen-bonding interaction of DDP dye with $\mathrm{GuHCl}$ in water

Table 1. Fluorescence lifetime decay analysis of DDP dye with $\mathrm{GuHCl}$ in water

\begin{tabular}{lllllllll}
\hline S.No & {$[\mathbf{M}]$} & $\mathbf{T}_{\mathbf{1}}(\mathbf{n s})$ & $\mathbf{T}_{\mathbf{2}}(\mathbf{n s})$ & $\mathbf{T}_{\mathbf{3}}(\mathbf{n s})$ & $\mathbf{B}_{\mathbf{1}} \%$ & $\mathbf{B}_{\mathbf{2}} \%$ & $\mathbf{B}_{\mathbf{3}} \%$ & $\boldsymbol{\chi}^{\mathbf{2}}$ \\
\hline 1 & 0.0 & 0.31 & 3.12 & 6.75 & 6 & 31 & 63 & 1.16 \\
2 & 1.0 & 0.18 & 1.41 & 4.15 & 9 & 20 & 70 & 1.21 \\
3 & 2.0 & 0.15 & 1.71 & 4.71 & 6 & 31 & 63 & 1.12 \\
4 & 3.0 & 0.17 & 1.71 & 4.61 & 4 & 31 & 64 & 1.23 \\
5 & 4.0 & 0.04 & 1.74 & 4.38 & 9 & 29 & 62 & 1.25 \\
6 & 5.0 & 0.02 & 1.09 & 3.82 & 7 & 29 & 64 & 1.13 \\
\hline
\end{tabular}

Our earlier studies on the fluorescence lifetime of DDP dye in the presence of amidealso exhibits a triexponential lifetime components which signifies that there exist a minimum of three different hydrophobic phase resulting in the formation of a new lifetime. A similar phenomenon was observed in the interaction of DDP dye with amides.

\subsection{Cyclic Voltammogram of DDP Dye- $\mathrm{GuHCl}$}

Electrochemical investigation of host (DDP dye) -guest $(\mathrm{GuHCl})$ interaction in aqueous solution were studied using cyclic voltammetry. DDP- GuHCl interaction resulted in increase in anodic peak current from DDP dye alone. Cyclic Voltammogram of $7.1 \times 10^{-4} \mathrm{M}$ DDP dye and DDP dye- $\mathrm{GuHCl}$ of different concentration ranging from $1.0 \mathrm{M}$ to $6.0 \mathrm{M}$ with $\mathrm{Ag} / \mathrm{AgCl}$ as reference electrode and $0.01 \mathrm{M} \mathrm{KCl}$ as supporting electrolyte at scan rate $0.1 \mathrm{Vs}^{-1}$ is shown in Figure 6. The current response just described that the shift in anodic peak potential towards less positive value indicate electrostatic binding (Bard \& Faulkener, 1980; Randles, 1948; Carter \& Bard, 1987; Carter, Rodriguez, \& Bard, 1989). The electrostatic force is may be due to hydrogen bonding formation in DDP dye with $\mathrm{GuHCl}$ was also correlated from emission spectra and EIS study. 


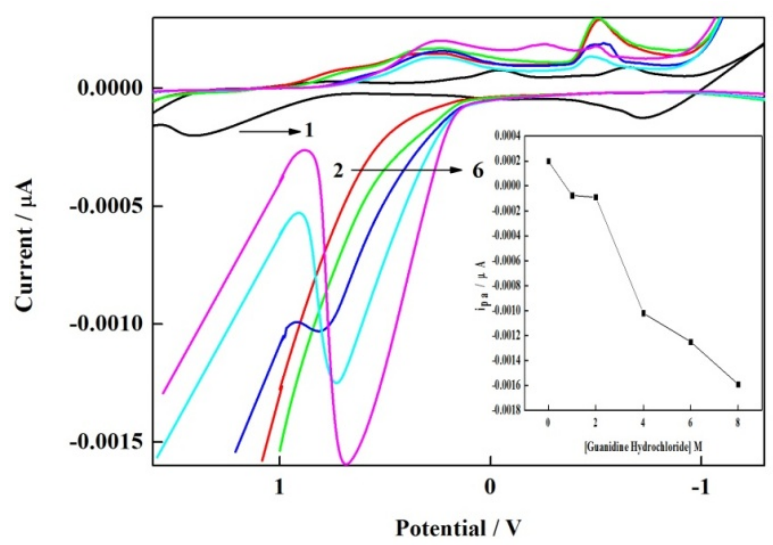

Figure 6. Cyclic Voltammogram of $7.1 \times 10^{-4} \mathrm{M}$ DDP dye and DDP dye- $\mathrm{GuHCl}$ with $\mathrm{Ag} / \mathrm{AgCl}$ as reference electrode and $0.01 \mathrm{M} \mathrm{KCl}$ as supporting electrolyte at scan rate $0.1 \mathrm{Vs}^{-1} .1$ ) DDP dye, 2) DDP dye $+1.0 \mathrm{M}$ GuHCl, 3) DDP dye + 2.0 M GuHCl, 4) DDP dye + 3.0 M GuHCl, 5) DDP dye + 4.0 M GuHCl, 6) DDP dye + $5.0 \mathrm{M} \mathrm{GuHCl}$.(Inset Figure: Langmuir adsorption isotherm of $7.1 \times 10^{-4} \mathrm{M}$ DDP dye- (x) Formamide, $1.0 \mathrm{M} \geq \mathrm{x}$ $\leq 8.0 \mathrm{M}$ from the relationship of $[\mathrm{GuHCl}] \mathrm{M} \mathrm{Vs} \mathrm{i}_{\mathrm{pc}} / \mu \mathrm{A}$ )

From inset of Figure 6 it is clear that the anodic peak currents of the DDP dye increased with increasing the concentrations of $\mathrm{GuHCl}$ as expected for Langmuir adsorption behaviour. For quantitative comparison of the binding constant, $\mathrm{K}$ between DDP dye with $\mathrm{GuHCl}$ was explored using the Langmuir equation (1) (Qin, Cai, Miao, Wang, \& Hu, 2014; Zhang \& Liu, 2011) as used by Qu.et al. (Liu, Xu, \& Chen, 2004; Qu, Li, \& Jiang, 1998).

$$
1 / \Delta \mathrm{I}_{\mathrm{p}}=1 / \Delta \mathrm{I}_{\mathrm{pmax}}+1 / \Delta \mathrm{I}_{\mathrm{pmax}} \times \mathrm{K} .1 /[\mathrm{GuHCl}]
$$

We assume, $\Delta \mathrm{I}_{\mathrm{p}}$ represent $\Delta \mathrm{I}_{\mathrm{p}}, \Delta \mathrm{I}_{\mathrm{pmax}}$ represent $\Delta \mathrm{I}_{\max }$, where $\Delta \mathrm{I}_{\mathrm{p}}=\mathrm{I}_{\mathrm{p} 0}-\mathrm{I}_{\mathrm{p}}$, where $\mathrm{I}_{\mathrm{p} 0}$ and $\mathrm{I}_{\mathrm{p}}$ represent the cathodic peak current in the absence and presence of urea. Theplot of $1 /$ [GuHCl] M Vs $1 / \mathrm{I}_{0}-\mathrm{I}$ is shown in Figure 7 is used for determination of binding constant, $\mathrm{K}$.

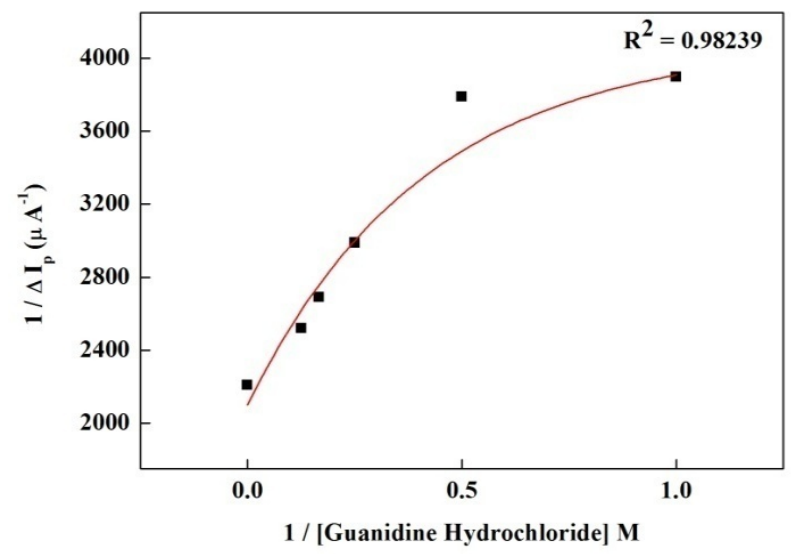

Figure 7. 1 / [GuHCl] M Vs $1 / \Delta \mathrm{I}_{\mathrm{p}}\left(\mu \mathrm{A}^{-1}\right)$ Binding plot for determination of Binding Constant, $\mathrm{K} .\left(\Delta \mathrm{I}_{\mathrm{p}}=\mathrm{I}_{\mathrm{p} 0}\right.$ and $\mathrm{I}_{\mathrm{p}}$ are the difference in peak currents before and after addition of $\mathrm{GuHCl}$ )

The binding plot derived from Langmuir equation clearly reveals that the binding of GuHCl with DDP dye does not form any complex. The nature of binding fit show there is no direct 1:1 but there exist more than one binding sites and also binding exist in different microenvironment in aqueous phase. The binding constant and Free energy were found to be $2.4 \times 10^{-3} \mathrm{~mol} \mathrm{~L}^{-1}, 32.76 \times 10^{3} \mathrm{~K} . \mathrm{J} \cdot \mathrm{mol}^{-1}$ respectively with regression co-efficient $\left(\mathrm{R}^{2}=\right.$ $0.98239)$. 


\subsection{Electrochemical Impendence Spectroscopy (EIS)}

A plot of z vs z' (Nyquist or cole-cole plots) taken over a wide frequency range $(50 \mathrm{~Hz}$ to $5 \mathrm{MHz})$ at room temperature and fitted with equivalent circuit as shown in supporting informationFigure 8 . An equivalent circuit of charge transfer capacitance $\mathrm{C}_{\mathrm{ct}}$ and charge transfer resistance, $\mathrm{R}_{\mathrm{ct}}$ was in parallel connection with resistance in series $R_{s}$. The number of environment involved in the charge transfer process is equal to the number of equivalent circuits. The capacitive behaviour of free dye with three equivalent circuits representing three environments as, i) a semicircle is observed at high frequencies, attesting the charge transfer at the electrode/solution interface, ii) a transition zone at intermediary frequencies, iii) a Warburg like line at low frequencies characteristic for diffusion process (Gayathri, Vasanthi, Vanjinathan, \& Kumaran, 2018).The R(CR) depicts the Randels circuit of an electrochemical cell which ensures the electrostatic mode of attraction between the dye and $\mathrm{GuHCl}$ molecule. On addition of $\mathrm{GuHCl}$, to DDP dye we observe that a charge transfer capacitance $\mathrm{C}_{\mathrm{ct}}$ and charge transfer resistance, $\mathrm{R}_{\mathrm{ct}}$ decrease with equivalent circuits is shown in inset of Figure 8.

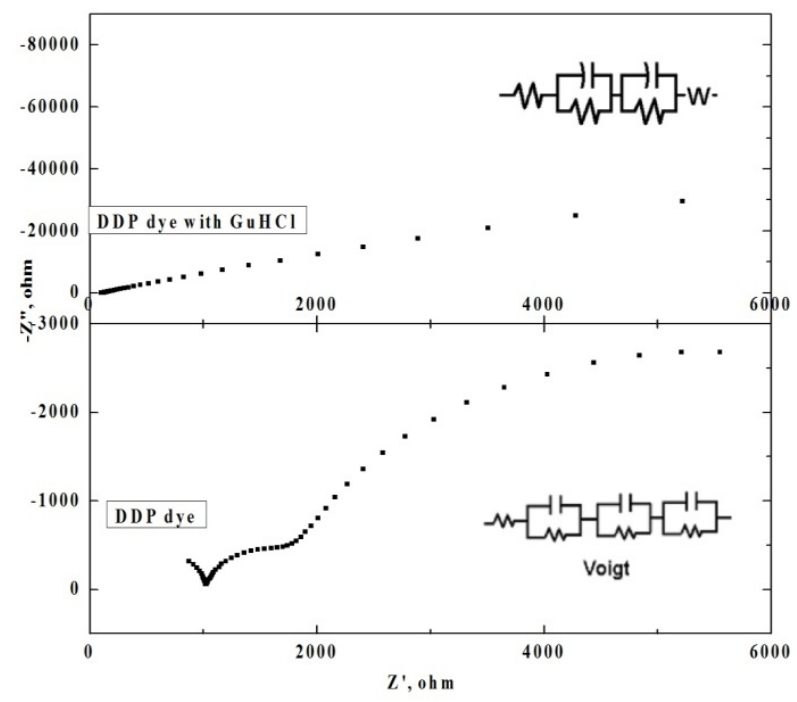

Figure 8. Electrochemical Impedance Spectroscopy of DDP dye in water in the absence and presence of GuHCl

The presence of Warburg or capacitive behaviour and the considerable decrease in resistance is the evidence for the formation of diffusion layer (Lupu, 2011) in the impedance spectra of dye with GuHCl. The existence of two equivalent circuits with Warburg as shown in Figure 7 implies that dye interaction with GuHCl exits in more than one microenvironment which is congruent with the result lifetime decay spectroscopy of DDP dye interaction with $\mathrm{GuHCl}$.

\section{Conclusions}

DDP dye interaction with $\mathrm{GuHCl}$ by various techniques like UV-Visible, fluorescence spectroscopy and cyclic voltammetry were employed as a tool in establishing the nature of interaction. These techniques are used to evaluate the binding nature as well as preferred mode of interaction of DDP dye in the presence of $\mathrm{GuHCl}$. The shift in peak potential and current of the DDP dye decreases with increasing concentration of GuHCl. This is attributed to electrostatic nature accompanied with hydrogen-bonding mode of interaction. The fluorescence enhancement on the addition of $\mathrm{GuHCl}$ which reveals that the excited state properties of DDP is influenced by change in microenvironment due to the presence of hydrogen-bonding moieties. Further, we establish that the interaction of DDP dye with $\mathrm{GuHCl}$ is also through electrostatic mode apart from hydrogen-bonding interaction. Electrochemical studies coupled with fluorescence spectral techniques provide a convenient tool to characterize the most possible binding nature of DDP dye(Host) with $\mathrm{GuHCl}$ (Guest) molecules.

\section{Supporting Information}

\section{Experimental setup of TSCPC}

Time-resolved fluorescence measurements- Time correlated single-photon counting (TCSPC) technique

The Fluorescence decay measurements of DDP dye with $\mathrm{GuHCl}$ were recorded using IBH time correlated 
single-photon counting spectrometer with microchannel plate photomultiplier tube (MCP-PMT) (Hamamatsu, R3809U) as detector. The data analysis was carried out by the software provided by IBH (DAS-6) which is based on reconvolution technique using iterative non-linear least square methods (Gayathri, Vasanthi, Vanjinathan, \& Kumaran, 2018). A detailed experimental setup and analysis is provided in the supporting information. A diode pumped millennia V CW laser (spectra physics) was used to pump the Ti-Sapphire rod in a Tsunami pico second-mode locked laser system (spectra physics). The 750nm (85 MHz) beam from the Ti-Sapphire laser was passed through a pulsepicker (spectra physics, GWU 23PS) to generate 4MHz pulses. The second harmonic output was generated by a flexible harmonic generator (spectra physics, GWU 23PS). A vertically polarized $375 \mathrm{~nm}$ laser was used to excite the sample. TCSPC is a digital technique, counts the photons, which are time correlated with the excitation pulse. The heart of the method is a time-to-amplitude converter (TAC).The fluorescence photons are collected at the magic angle $\left(54.7^{\circ}\right)$ to avoid the distortions due to the rotational polarization. The instrument response function is around $50 \mathrm{ps}$. The measured decay is the convolution of the true fluorescence decay, excitation function and the instrument response function which results in the fluorescence kinetics parameters like lifetime $(\tau)$ and relative amplitude $(\mathrm{A})$. The data analysis was carried out by the software provided by IBH (DAS-6) which is based on reconvolution technique using iterative non-linear least square methods. The reconvolution is preceded by the series of iterations until a chi-square is reduced. The quality of fit is normally identified by the reduced $\chi^{2}$, weighed residual and autocorrelation function of the residuals.

\section{Acknowledgements}

The authors are thankful to Prof. P. Ramamurthy and Dr. C. Selvaraju of National Centre for Ultrafast Processes, University of Madras, Chennai-600 113, India, for permitting us to avail the fluorescence facilities. S.G and R.K thank Dr. S.G. Srivatsan and Dr Hazra Partha, IISER Pune, for availing TCSPC Facilities. The authors thank Shri Ashok Kumar Mundra, the Secretary, Dr. R. Ganesan Principal, and the Management of Dwaraka Doss Goverdhan Doss Vaishnav College, Arumbakkam, Chennai-600 106 for their support and encouragement.

\section{Conflict of interests}

The authors declare that there is no conflict of interests regarding the publication of this paper.

\section{References}

Bard, A. J.,\& Faulkener, L. R. (1980).Electrochemical Methods. Fundamentals And Applications, New York.

Benesi, H.,\& Hildebrand, J. H. A. (1949). Spectrophotometric Investigation of the interaction of Iodine with Aromatic hydrocarbons. Journal of American Chemical Society, 71(8), 2703.

Carter, M.T.,\& Bard, A.J. (1987). Voltammetric studies of the interaction of tris(1,10-phenanthroline)cobalt(III) with DNA.Journal of American Chemical Society, 109(24), 7528-7530.

Carter, M.T., Rodriguez, M., \&Bard, A.J. (1989). Voltammetric studies of the interaction of metal chelates with DNA. 2. Tris-chelated complexes of cobalt(III) and iron(II) with 1,10-phenanthroline and 2,2'-bipyridine. Journal of American Chemical Society, 111(24), 8901-8911.

Castellino, F.J.,\& Barker, R. (1968). The denaturing effectiveness of guanidinium, carbamoylguanidinium, and guanylguanidinium salts. Biochemistry, 7(11), 4135-4138.

Cox, R.A. (1968). The use of guanidinium chloride in the isolation of nucleic acids. In L. Grossman \&K. Moldave(Eds.),Methods In Enzymology (Vol. 12, pp. 120-129). Academic, New York.

Garza-Ramos, G., Fernandez-Velasco, D. A., Ramirez, L., Shoshani, L., ...\&\& Gómez-Puyou, A. (1992). Enzyme activation by denaturants in organic solvent systems with a low water content.European Journal of Biochemistry, 205(2), 509-517.

Gayathri, S., Vasanthi, R., Vanjinathan, M., \&Kumaran, R. (2018). Photophysical and Electrochemical studies of 4-dicyanomethylene 2,6-dimethyl-4H-pyran (DDP) dye with amides in water.Journal of Fluorescence, 28(6), 1379-1388.

Hammond, P. R. (1979). Laser dye DCM, Its Spectral Properties, Synthesis and Comparison with Other Dyes in the Red. Optics Communication, 29(3), 331-333.

Hedwig, G.R., Lilley, T.H., \&Linsdell, H. (1991). Calorimetric and volumetric studies of the interactions of some amides in water and in $6 \mathrm{~mol} \mathrm{dm} \mathrm{dm}^{-3}$ aqueous guanidinium chloride. Journal of Chemical Society Faraday Transactions, 87(18), 2975-2982.

Hsieh, B. Y.,\& Chen, Y. (2007). Synthesis, Photophysics, and Electroluminescence of Copolyfluorenes Containing DCM Derivatives. Macromolecules, 40(25), 8913-8923. 
Katz, S. (1968). Partial molar volume and conformational changes produced by the denaturation of albumin by guanidine hydrochloride. Biochimica et Biophysica Acta, 154(3), 468-477.

Kawahara, K., \& Tanford, C. (1966). Viscocity and density of aqueous solution of urea and guanidine hydrochloride. Journal of Biological Chemists, 241(13), 3228-3232. Retrieved from http://www.jbc.org/content/241/13/3228

Kumaran, R.,\& Ramamurthy, P. (2006). PET suppression of Acridinediones by urea derivatives in water and methanol.Journal of Physical Chemistry B, 110(15), 23783-23789.

Kumaran, R.,\& Ramamurthy, P. (2010). Photophysical studies of PET based Acridinedione dyes with globular protein: Bovine Serum Albumin (BSA). Journal of Luminescence., 130(7), 1203-1210.

Kumaran, R., Varalakshmi, T., \&Padma Malar, E. J. (2010). Ramamurthy, P. Photophysical Studies on the Interaction of Acridinedione Dyes with Universal Protein Denaturant: Guanidine Hydrochloride. Journal of Fluorescence., 20(5), 993-1002.

Liu, S.Q., Xu, J. J., \& Chen, H. Y. (2004). A reversible adsorption-desorption interface of DNA based on nano-sized zirconia and its application. Colloids Surface. B: Biointerfaces, 1/36(3-4), 155-9.

Lupu, S. (2011). Electrochemical impedance measurements on prussian blue films deposited on platinum electrodes.U.P.B. Scientific Bulletin., Series B, 73(1), 85-96. Retrieved from https://www.scientificbulletin.upb.ro/rev_docs_arhiva/full35322.pdf

Ma, Y.Z., \& Tsou, C.L. (1991). Comparison of the activity and conformation changes of lactate dehydrogenase H. during de- naturation by guanidinium chloride.Biochemistry Journal, 277(1), 207- 211.

Makhatadze, G.I., \&Privalov, P.L. (1992). Protein interactions with urea and guanidinium chloride: A calorimetric study. Journal of Molecular Biology, 226(2), 491-505.

Marason, E. G. (1981). Laser dyes DCM: CW, Synchronously Pumped, Cavity Pumped and Single-Frequency Performance. Optics Communication, 37(1), 56-58.

Meyer, M.,\& Mialocq, J. C. (1987). Ground State and Singlet Excited Stat of Laser Dye DCM: Dipole Moments and Solvent Induced Spectral Shifts.Optics Communication, 64(3), 264-268.

Michnik, A., \&Sulkowska, A. (1997). Hydrogen-bonded interactions in alkylurea-and amide- $\mathrm{D}_{2} \mathrm{O}$-gunanidine. $\mathrm{HCl}$ systems. Journal of Molecular Structure, 410-411(1), 17-21.

Miggiano, A. D., Mordente, A., Pischiutta, M. G., Martorana, G. E., \&Castelli, A. (1987). Early conformational changes and activity modulation induced by guanidinium chloride on intestinal alkaline phosphatase. Biochemistry Journal, 248(2), 551-556.

Moosavi-Movahedi, A.A., Naderi, G.A., \& Farzami, B. (1994). The denaturation behaviour of calmodulin in sodium n-dodecyl sulphate, dodecyl trimethyl ammonium bromide, guanidine hydrochloride and urea. Thermochimica Acta, 239, 61-71.

Morjana, N.A., McKeone, B.J., \&Gilbert, H.F. (1993). Guanidine hydrochloride stabilization of a partially unfolded intermediate during the reversible denaturation of protein disulfide isomerase. Proceeding of the National Academy of Sciences of the United States of America, 90(6), 2107-2111.

Qin, D. A., Cai, X. Q., Miao, Q., Wang, Z. H.,\&Hu, M. L. (2014). Cyclic Voltammetry and Molecular Docking Study of the Interactions of Two Derivatives of 5-fluorouracil with DNA. International Journal of $\begin{array}{lllll}\text { Electrochemical } & \text { Science, } & 9(4), & 1608-1620 . & \text { Retrieved }\end{array}$ http://www.electrochemsci.org/papers/vo19/90401608.pdf

Qu, F., Li, N.Q., \&Jiang, Y.Y. (1998). Electrochemical studies of NiTMpyP and interaction with DNA. Talanta, 45(5), 787-93.

Randles, J. E. B. (1948). A cathode ray polarograph. Part II.-The current-voltage curves. Transactions of Faraday Society, 44, 327-338.

Shoshani, L.,Darszon, A.,Tijena de Gomez-puyou, M.,\& Gomez-puyou, A. (1994). Activity and fluorescence changes of lactate dehydrogenase induced by guanidine hydrochloride in reverse micelles.European Journal of Biochemistry, 221(3), 1027-1032.

Sowntharya, C., Gayathri, S., Dhenadayalan, N., Vasanthi, R., Vanjinathan, M., \&Kumaran, R. (2017). Photophysical studies of a Food Hydrocolloid, Gum Arabic with Resorcinol based Acridinedione dyes in water. Journal of Photochemistry and Photobiology A, 341, 78-86. 
Srividya, N., Ramamurthy, P., Shanmugasundaram, P., \&Ramakrishna, V.T. (1996). Synthesis, characterisation and electrochemistry of some acridine-1, 8- dione dyes. Journal of Organic Chemistry, 61(15), 5083-5089.

Strambini, G. B., \& Gonnelli, M. (1986). Effect of urea and guanidine hydrochloride on the activity and dynamical structure of equine liver alcohol dehydrogenase.Biochemistry, 25(9), 2471-2476.

Woods, L. L. (1958). Some Further Reactions of 2, 6-Dimethyl-4-pyrone. Journal of American Chemical Society, 80(6), 1440-1442.

Zarins, E., Jubels, J., \&Kokars, V. (2011). Synthesis of red luminescent non symmetric styryl-4Hpyran-4-ylidene fragment containing derivatives for organic light-emitting diodes. Advanced Materials Research, 222, 271-274.

Zettl meissl, G., Rudolph, R., \&Jaenicke, R. (1982). Rate determining folding and association reactions on the reconstitution pathway of porcine skeletal muscle lactic dehydrogenase after denaturation by guanidine hydrochloride.Biochemistry, 21(17), 3946-3950.

Zhang, K. J.,\&Liu, W. Y. (2011). Investigation of the Electrochemical Interaction Behavior of DNA with 5-Fluorouracil Derivatives. International Journal of Electrochemical Science, 6(5), 1669-1678. Retrieved from http://www.electrochemsci.org/list11.htm

\section{Copyrights}

Copyright for this article is retained by the author(s), with first publication rights granted to the journal.

This is an open-access article distributed under the terms and conditions of the Creative Commons Attribution license (http://creativecommons.org/licenses/by/4.0/). 